\title{
Correlations in impact-parameter space in saturation models
}

\section{S. Munier*}

Centre de physique théorique, École Polytechnique, CNRS, 91128 Palaiseau, France

E-mail: Stephane.Munierdcpht.polytechnique.fr

\begin{abstract}
In order to get an estimate of the homogeneity of the distribution of matter in a fast hadron, we compute the correlation of the saturation scales between different impact parameters. We find that these correlations are quite strong: The saturation scale is nearly uniform in a wide domain around each point in impact-parameter space. We provide analytical expressions for the correlations, which are supported by numerical simulations. Although the numerical calculations are done for specific saturation models which are obtained from QCD after drastic simplifications, we expect our analytical formulas to be correct for full QCD in asymptotic limits, since their derivation requires only a few general assumptions.
\end{abstract}

XVIII International Workshop on Deep-Inelastic Scattering and Related Subjects, DIS 2010 April 19-23, 2010

Firenze, Italy

\footnotetext{
*Speaker.
} 


\section{Introduction}

Phenomenological models for the very high-energy regime of QCD where saturation effects become important (see Ref. [1] for a recent review) are usually built on some parametrization of the elastic dipole-hadron scattering amplitude $T(y, r, b)$ which is a function of the rapidity $y$ of the scattering, ${ }^{1}$ of the size $r$ of the dipole, and of the impact parameter $b$. This amplitude is then related to the observables through appropriate convolutions with the wave functions that describe the incoming objects. In the simplest of these models, due to Golec-Biernat and Wüsthoff [纤], the dipole amplitude is assumed to have the form

$$
T(r, y, b)=1-e^{-r^{2} Q_{s}^{2}(y, b) / 4},
$$

where the momentum scale $Q_{s}$, called the saturation momentum, is parametrized as

$$
Q_{s}^{2}(y, b)=1 \mathrm{GeV}^{2} \times \theta(R-b) e^{\lambda\left(y-y_{0}\right)} .
$$

The constants $R, \lambda$ and $y_{0}$ are determined from a fit to the inclusive deep-inelastic scattering data. The spatial distribution of matter in the plane transverse to the collision axis is encoded in the $b$ dependence of the saturation momentum. The $\theta$-function used by Golec-Biernat and Wüsthoff is sometimes changed to a smoother distribution in such a way that the model be also able to describe semi-inclusive diffractive data. In any case, the fluctuations between different points in transverse space are completely neglected in all these models. Note that this may not be a problem for standard phenomenology since most of the observables in deep-inelastic scattering probe one single point in impact-parameter space in each event. But clearly, independently of phenomenology, we would like to understand better how the matter is distributed in a fast hadron.

We shall first explain why fluctuations of the parton densities are expected between different impact parameters, then we shall provide a heuristical discussion of the form of these fluctuations, for which we have been able to write a parameter-free formula valid in some asymptotic limit.

\section{Picture of a fast hadron/nucleus}

Let us consider a fast hadron or nucleus probed by a color dipole of size $r$ (which may be seen as a component of a virtual photon of virtuality $Q \sim 1 / r$ ) at very high rapidity $y$. We go to a frame in which the probing dipole is almost at rest and we require that the impact parameter be some fixed $b$ (see Fig. 1). The scattering probability $T$ is roughly proportional to the local density $n$ of partons in the corresponding phase-space cell: $T(r, y, b) \simeq \alpha_{s}^{2} n(r, y, b)$. It proves useful to see $T$ as a probability of interaction between the dipole and a fixed configuration of partons: The physical amplitude measured in experiments is then $T$ averaged over events (i.e. over the partonic configurations; see Ref. [3] for a review). If the rapidity is high enough, we know that at each $b$, $T$ has the shape of a front connecting 1 (black or saturated regime) for $r \gg 1 / Q_{s}(y, b)$ to 0 (color transparent or dilute regime) for $r \ll 1 / Q_{s}(y, b)$. The saturation momentum $Q_{s}(y, b)$ determines the transition. It grows exponentially with $y$, which means that the position of the wave front moves linearly along the axis $\log \left(1 / r^{2}\right)$ when the rapidity increases. It was first conjectured [3] and then

\footnotetext{
${ }^{1}$ Throughout our discussion, $y$ is actually the rapidity multiplied by the factor $\bar{\alpha}=\alpha_{s} N_{c} / \pi$.
} 

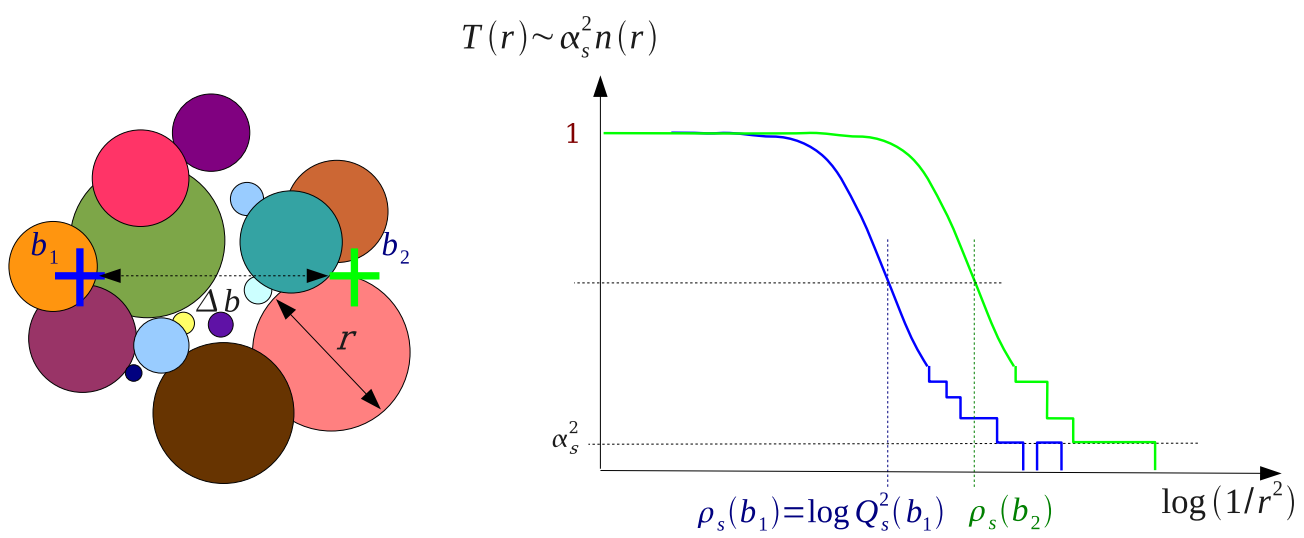

Figure 1: Schematic picture of a fast-moving hadron (left; the colored disks represent partons) and scattering amplitude as a function of the size $r$ of the probing dipole at two impact parameters $b_{1}, b_{2}$ for a fixed rapidity $y$ (right).

checked numerically [ [ $₫$ ] that to a good approximation, at each point in impact-parameter space, the only variable relevant to the evolution of the amplitude $T$ with the rapidity $y$ is $\log \left(1 / r^{2}\right)$.

In the regions in which $T \sim \alpha_{s}^{2}$, few partons are probed, hence the further evolution of $T$ with the rapidity $y$ is stochastic. If $T \gg \alpha_{s}^{2}$ instead, many partons populate that phase-space region, and the evolution of $T$ is of deterministic nature: A mean-field approximation of the QCD evolution can be taken. Thus for values of $r$ of the order of the inverse saturation momentum, $T$ has the shape of a smooth (deterministic) curve traveling towards smaller values of $r$. However, because of the fluctuations in the tail of the front, $Q_{s}$ is a stochastic variable for the rapidity evolution. Fluctuations in the dilute region of phase space propagate towards the dense region and affect the saturation momentum typically after an additional evolution over $\Delta y \sim \log ^{2}\left(1 / \alpha_{s}^{2}\right)$ units of rapidity. They result in a random diffusion of $\rho_{s} \equiv \log Q_{s}$ of variance $\left\langle\rho_{s}^{2}\right\rangle_{c} \sim D y$, where $D$ can be computed from QCD [3].

These fluctuations determine a dispersion of $\rho_{s}$ from event to event. But stochasticity is also expected to manifest itself by differentiating the points say $b_{1}$ and $b_{2}$ in impact-parameter space, creating a dispersion of $\rho_{s}$ in the transverse plane. In order to characterize these fluctuations, we shall now compute the correlator $\sigma_{12}^{2} \equiv\left\langle\left(\rho_{s}\left(b_{1}\right)-\rho_{s}\left(b_{2}\right)\right)^{2}\right\rangle$ at fixed $y$.

\section{How correlations may occur: heuristic discussion and analytical formulas}

Let us examine how correlations between two points in transverse space $b_{1}$ and $b_{2}$ may build up. We define $\Delta b=\left|b_{2}-b_{1}\right|$. If $\Delta b<1 / Q_{s}$ ( $Q_{s}$ is the saturation momentum at either $b_{1}$ or $\left.b_{2}\right)$, then obviously $Q_{s}\left(b_{1}\right)=Q_{s}\left(b_{2}\right)$ and $\sigma_{12}^{2}=0$. If $\Delta b>1 / Q_{s}$ instead, then the evolution around the impact parameter $b_{1}$ can influence the evolution around $b_{2}$ only if a parton at $b_{1}$ splits into a parton of size of the order of $\Delta b$. But the saturation of the density of partons of sizes larger than $1 / Q_{s}$ disfavors such splittings. Hence we may think that the evolutions decouple as soon as the saturation radius $1 / Q_{s}$ becomes smaller than $\Delta b$. Assume that this happens at rapidity $y_{0}$ : Then for $y<y_{0}$, $\sigma_{12}^{2}=0$, and for $y>y_{0}, \sigma_{12}^{2} \simeq\left\langle\rho_{1}^{2}\right\rangle_{c}+\left\langle\rho_{2}^{2}\right\rangle_{c} \sim 2 D\left(y-y_{0}\right)$. One may fix the rapidity $y$ and vary the distance $\Delta b$ instead: Then $\sigma_{12}^{2} \sim 2 D \log \left(\Delta b Q_{s}\right) / \chi^{\prime}\left(\gamma_{0}\right)$ for $\log \left(\Delta b Q_{s}\right)>0$ (see the dotted line in Fig. 2), which suggests that the characteristic distance scale for the correlations in the transverse 


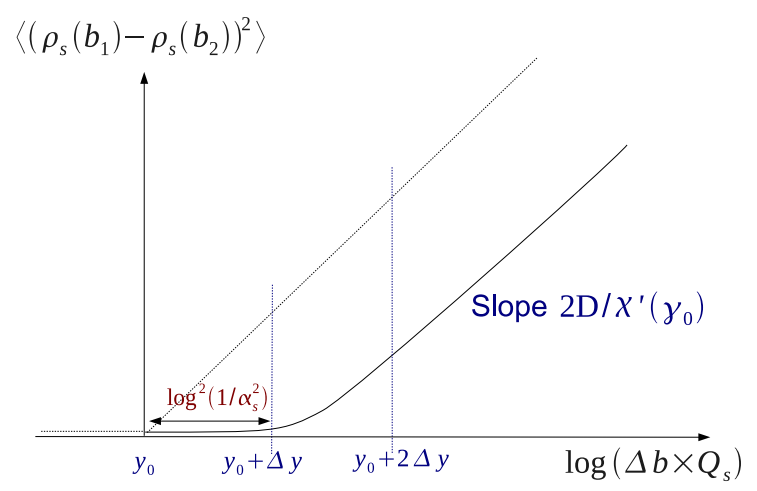

Figure 2: Sketch of the correlations as a function of the logarithm of the distance $\Delta b \equiv\left|b_{1}-b_{2}\right|$ in impactparameter space scaled by $1 / Q_{s}$. For $\Delta b Q_{s}>1$, the points $b_{1}$ and $b_{2}$ are statistically independent. The dotted line represents what one would naively expect if fluctuations affected the saturation scale as soon as $\Delta b Q_{s}>1$. ( $D$ is the diffusion coefficient of $\rho_{s}$ for a single front, namely $D \sim\left\langle\rho_{s}^{2}\right\rangle_{c} / y$ ). The continuous line takes into account the delay induced by the propagation of the fluctuations, which results in an effective persistence of the correlations.

plane is $1 / Q_{s} \cdot \chi\left(\gamma_{0}\right)$ is a particular eigenvalue of the BFKL kernel $\chi$, and $\chi^{\prime}\left(\gamma_{0}\right)$ the asymptotic rate of change of $\left\langle\rho_{s}\right\rangle$ with the rapidity [3] .

However, this is not yet the correct answer. Indeed, as recalled before, for fluctuations to be able to differentiate $b_{1}$ and $b_{2}, \Delta y \sim \log ^{2}\left(1 / \alpha_{s}^{2}\right)$ extra units of rapidity are needed after the rapidity $y_{0}$ at which $\Delta b Q_{s}\left(y_{0}\right)=1$. Hence the effective decoupling of the saturation momenta is expected later in rapidity, or for larger distances $\Delta b$. The correlations would persist over distances $\Delta b \sim e^{c \log ^{2}\left(1 / \alpha_{s}^{2}\right)} / Q_{s}$ (see the full line in the sketch of Fig. 2).

Extending the phenomenological theory for stochastic fronts developed in Ref. [5], we are able to fully compute the correlator $\sigma_{12}^{2} \equiv\left\langle\left(\rho_{s}(b)-\rho_{s}(b+\Delta b)\right)^{2}\right\rangle$. One way of writing the result is [6]

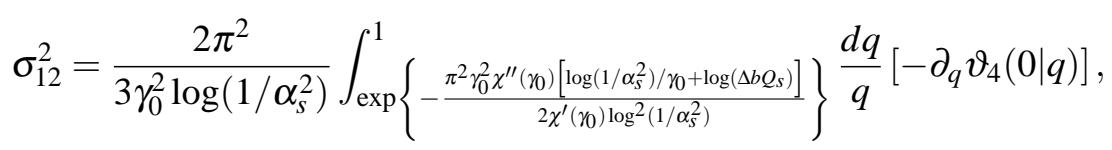

where $\vartheta_{4}$ is a particular Jacobi theta function. The interesting limiting behaviors read

$$
\sigma_{12}^{2} \sim \begin{cases}\frac{2 \pi^{4} \chi^{\prime \prime}\left(\gamma_{0}\right) \log \left(\Delta b Q_{s}\right)}{3 \chi^{\prime}\left(\gamma_{0}\right) \log \left(1 / \alpha_{s}^{2}\right)} & \text { for } \log \left(\Delta b Q_{s}\right) \gg \log ^{2}\left(1 / \alpha_{s}^{2}\right) \\ \frac{4}{3 \gamma_{0}^{3}} \sqrt{\frac{2 \pi^{3} \chi^{\prime}\left(\gamma_{0}\right)}{\chi^{\prime \prime}\left(\gamma_{0}\right) \log \left(\Delta b Q_{s}\right)}} \exp \left(-\frac{\chi^{\prime}\left(\gamma_{0}\right) \log ^{2}\left(1 / \alpha_{s}^{2}\right)}{2 \gamma_{0}^{2} \chi^{\prime \prime}\left(\gamma_{0}\right) \log \left(\Delta b Q_{s}\right)}\right) & \text { for } \log \left(1 / \alpha_{s}^{2}\right) \ll \log \left(\Delta b Q_{s}\right) \ll \log ^{2}\left(1 / \alpha_{s}^{2}\right) .\end{cases}
$$

Comparing the expression of $\sigma_{12}^{2}$ in the large $\Delta b$ limit to the variance $D y$ of $\rho_{s}$, we find that $\sigma_{12}^{2}$ is actually equal to $2 D \log \left(\Delta b Q_{s}\right) / \chi^{\prime}\left(\gamma_{0}\right)$ for $\operatorname{large} \log \left(\Delta b Q_{s}\right)$. From the second limiting expression, it is obvious that $\sigma_{12}^{2}$ is close to zero for $\log \left(\Delta b Q_{s}\right) \ll \log ^{2}\left(1 / \alpha_{s}^{2}\right)$.

In order to check these expressions, we performed numerical simulations of models which possess the main characteristics of the QCD evolution while being simple enough to allow for robust Monte Carlo simulations (see Ref. [6] for details). We found a perfect matching with the parameter-free analytical result (3.1) in the limit $\log \left(1 / \alpha_{s}^{2}\right) \gg 1$. For larger and more realistic values of $\alpha_{s}$, the persistence of the correlations is still seen in the numerical simulations, but some parameters should be modified in the analytical expressions and tuned to account for our lack of 


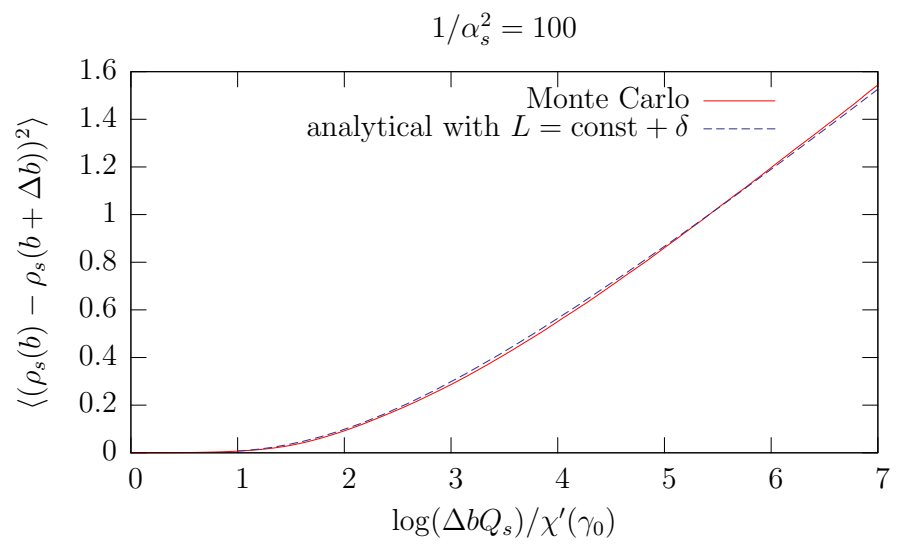

Figure 3: Comparison of a numerical Monte Carlo simulation and our analytical formula. The constant in the parameter $L$ (see Ref. [6] for the definitions of $L$ and $\delta$ ), which should be equal to $\log \left(1 / \alpha_{s}^{2}\right) / \gamma_{0}$ for very small $\alpha_{s}$, has been shifted by a phenomenological constant. Once this is done, we get a very good agreement between the two calculations.

understanding of subleading corrections important for finite $\log \left(1 / \alpha_{s}^{2}\right)$. We show such a calculation for $\alpha_{s}=0.1$ in Fig. 3, compared to a variant of Eq. (3.1).

\section{Conclusion and outlook}

The main result of our work is that the characteristic distance of the correlations in the transverse plane is not $1 / Q_{s}$ as one would naively expect, but rather $\exp \left[c \log ^{2}\left(1 / \alpha_{s}^{2}\right)\right] / Q_{s}(c$ being a known constant), which is parametrically much larger than $1 / Q_{s}$. Our results are valid for large $\log \left(1 / \alpha_{s}^{2}\right)$, and for distances $\Delta b$ much smaller than the typical confinement scale $1 / \Lambda_{\mathrm{QCD}}$.

The goal of our work was to understand the fundamentals of the QCD dynamics in transverse space, without thinking a priori of any application to phenomenology. Let us however note that recently, a diffractive deep-inelastic scattering observable was proposed that would directly probe the correlations which we have computed [7]. (A calculation of these correlations in the framework of the B-JIMWLK formalism [1] which a priori neglects the fluctuations discussed in this paper was also performed.) Also, these correlations may play an important role in heavy-ion collisions.

\section{References}

[1] F. Gelis, E. Iancu, J. Jalilian-Marian and R. Venugopalan, arXiv:1002.0333 [hep-ph].

[2] K. J. Golec-Biernat, M. Wüsthoff, Phys. Rev. D59 (1999) 014017.

[3] S. Munier, Phys. Rept. 473 (2009) 1.

[4] S. Munier, G. P. Salam, G. Soyez, Phys. Rev. D78 (2008) 054009.

[5] E. Brunet, B. Derrida, A. H. Mueller and S. Munier, Phys. Rev. E73 (2006) 056126.

[6] A. H. Mueller and S. Munier, Phys. Rev. D81 (2010) 105014.

[7] C. Marquet and H. Weigert, arXiv:1003.0813 [hep-ph]. 